\title{
Prognostic Model of Typical Complications Caused by Transcatheter Aortic Valve Replacement
}

\author{
DOI: $10.17691 / \mathrm{stm} 2020.12 .2 .03$
}

Received July 25, 2019

E.A. Ovcharenko, PhD, Head of Laboratory, Department of Experimental and Clinical Cardiology;

K.U. Klyshnikov, Researcher, Department of Experimental and Clinical Cardiology;

V.I. Ganyukov, MD, DSc, Head of Laboratory, Department of Multifocal Atherosclerosis;

A.A. Shilov, MD, PhD, Senior Researcher, Department of Multifocal Atherosclerosis;

I.E. Vereshchagin, Junior Researcher, Department of Multifocal Atherosclerosis;

I.N. Sizova, MD, PhD, Senior Researcher, Department of Cardiovascular Disease Diagnostics;

R.S. Tarasov, MD, DSc, Head of Laboratory, Department of Multifocal Atherosclerosis;

L.S. Barbarash, MD, DSc, Professor, Member of the Russian Academy of Sciences, Chief Researcher

Research Institute for Complex Issues of Cardiovascular Diseases, 6 Sosnovy Blvd, Kemerovo, 650002, Russia

The aim of the study was to develop a prognostic model based on statistical discriminant analysis to assess the risk of postoperative disturbance of cardiac conduction and paraprosthetic regurgitation after transcatheter aortic valve replacement.

Materials and Methods. Clinical data of 10 patients implanted with CoreValve ${ }^{\mathrm{TM}}$ prostheses (Medtronic Inc., USA) were used to develop prognostic models. To that end, we analyzed changes in hemodynamic and functional parameters provided by echocardiography in the pre- and postoperative periods.

Results. We observed significant positive changes in the severity of left ventricular myocardial hypertrophy; on the contrary, volume indicators did not significantly change, which might be associated with the concentric type of left ventricular hypertrophy. The discriminant analysis made it possible to determine major (preoperative) morphological and functional indicators associated with the two most common complications of the procedure: left bundle branch block and paraprosthetic regurgitation. Left ventricular posterior wall thickness, interventricular septal thickness, left atrium dimension, and myocardial mass are the critical factors that determine the development of these complications.

Conclusion. In the prognostic model, the proposed weighting coefficients allow one to assess the risk of postoperative complications; however, the presence of false-positive results requires further refinement of these coefficients within the linear equation.

Key words: transcatheter aortic valve replacement; cardiac conduction disturbances; paraprosthetic regurgitation; discriminant analysis.

\section{Introduction}

Transcatheter aortic valve replacement (TAVR) is becoming the gold standard for treating severe aortic stenosis in patients with contraindications for surgical aortic valve replacement (SAVR) [1]. In the recent decade, a series of randomized clinical trials comparing TAVR and SAVR were performed. In those, mortality and incidence of stroke were found comparable between these surgical treatments; in addition, significantly fewer complications were associated with TAVR [2, 3]. This progress can be explained by the increasing experience of interventional cardiologists, the optimized design of transcatheter valve prostheses and their delivery systems, and the wider use of three-dimensional (3D) imaging, including multispiral computed tomography (MSCT) at the stage of TAVR planning. All these factors contributed to the expansion of indications for using of TAVR technology in patients with medium and low surgical risk [4] and in patients with a bicuspid valve
$[5,6]$. Nevertheless, despite the rapid development of this technology, complications typical of this procedure remain: first of all, disturbances of cardiac conduction and paraprosthetic regurgitation [7]. An integrated approach to preoperative planning of TAVR that incorporates morphological and functional analysis can become a valuable tool for reducing the incidence of complications. We, therefore, analyzed the anatomical and hemodynamic changes in patients before and after the TAVR procedure.

The aim of the study was to develop a prognostic model based on statistical discriminant analysis to assess the risk of postoperative disturbance of cardiac conduction and paraprosthetic regurgitation after transcatheter aortic valve replacement.

\section{Materials and Methods}

Clinical data. A single-center retrospective study was conducted from August 2014 to November 2018 at the

Corresponding author: Kirill U. Klyshnikov, e-mail: KlyshnikovK@gmail.com 
Research Institute for Complex Issues of Cardiovascular Diseases (Kemerovo). We analyzed a number of clinical parameters in 10 patients undergoing TAVR procedures (CoreValve ${ }^{\mathrm{TM}}$ bioprosthesis; Medtronic Inc., USA) for aortic stenosis without hemodynamically significant regurgitation. Transfemoral access was used for the catheterization. Additional data were obtained by transthoracic echocardiography (TTE) before and after TAVR using a Vivid 7 Dimension ultrasound diagnostic scanner (General Electric, USA) and a sector phased M4S-RS sensor (1.5-3.6 MHz). The following indicators were studied: end-diastolic diameter (EDD), endsystolic diameter (ESD), ejection fraction (EF), enddiastolic volume (EDV), end-systolic volume (ESV), left atrium (LA) dimension, right ventricular (RV) dimension, interventricular septal (IVS) thickness, left ventricular posterior wall (LVPW) thickness, ascending aorta diameter, stroke volume (SV), myocardial mass (MM), and the maximal trans-prosthesis gradient (Pmax). We analyzed coded patient records that specified the diagnosis, clinical and demographic parameters, surgical risks according to EuroSCORE II, etc. The study was conducted in accordance with the Helsinki Declaration (2013) and approved by the Ethics Committee of the Research Institute for Complex Issues of Cardiovascular

Table 1

Clinical and demographic characteristics of the patients

\begin{tabular}{|c|c|}
\hline Characteristic & Value \\
\hline Quantity (abs. number) & 10 \\
\hline $\begin{array}{l}\text { Sex (abs. number } / \% \text { ): } \\
\text { male } \\
\text { female }\end{array}$ & $\begin{array}{l}5 / 50 \\
5 / 50\end{array}$ \\
\hline Age (years) ${ }^{*}$ & $79.5[67 ; 72.25 ; 80.75 ; 84]$ \\
\hline The maximum trans-prosthesis gradient $(\mathrm{mm} \mathrm{Hg})^{*}$ & $77.5[65.0 ; 74.0 ; 101.0 ; 115.0]$ \\
\hline Prior transdermal coronary interventions (abs. number $/ \%$ ) & $3 / 21.4$ \\
\hline Left ventricular ejection fraction $(\mathrm{ml})^{*}$ & $63[52 ; 56 ; 69 ; 71]$ \\
\hline Angina pectoris I-IV FC (abs. number/\%) & $4 / 40.0$ \\
\hline Ischemic heart disease (abs. number $/ \%$ ) & $8 / 80.5$ \\
\hline Pulmonary hypertension (abs. number/\%) & $7 / 70.0$ \\
\hline Surgical risk by the EuroSCORE II* & $2.9[2.3 ; 2.8 ; 3.6 ; 7.5]$ \\
\hline $\begin{array}{l}\text { Concomitant diseases (abs. number/\%): } \\
\text { arterial hypertension } \\
\text { chronic lower limb ischemia } \\
\text { significant stenosis of the internal carotid artery } \\
\text { history of acute cerebrovascular accident } \\
\text { diabetes } \\
\text { chronic kidney disease above stage III } \\
\text { chronic obstructive pulmonary disease } \\
\text { chronic renal failure }\end{array}$ & $\begin{array}{l}9 / 90.0 \\
1 / 10.0 \\
2 / 20.0 \\
1 / 10.0 \\
4 / 40.0 \\
3 / 30.0 \\
3 / 30.0 \\
2 / 20.5\end{array}$ \\
\hline $\begin{array}{l}\text { Sizes of CoreValve }{ }^{\mathrm{TM}} \text { (abs. number } / \% \text { ): } \\
26 \mathrm{~mm} \\
29 \mathrm{~mm}\end{array}$ & $\begin{array}{l}5 / 50.0 \\
5 / 50.0\end{array}$ \\
\hline Post-surgery follow up (years), $M \pm m$ & $0.57 \pm 0.25$ \\
\hline
\end{tabular}

* Data are presented as Me (min; 25; 75; max).
Diseases. Informed consent was obtained from each patient.

The prognostic model. To build a prognostic model based on TTE-revealed hemodynamic and functional changes caused by TAVR, a discriminant analysis with stepwise inclusion of quantitative variables was performed. Given the prognostic nature of discriminant analysis, preoperative TTE results were taken as input data. Two "soft" endpoints served as a grouping variable - the presence of a left bundle branch block (LBBB) and the presence of paraprosthetic regurgitation after the valve implantation. The quality of the analysis was evaluated by the significance level $p<0.05$, by the square of the Mahalanobis distance between two groups, and by re-classification of the available data sample using the obtained linear equation of the discriminant function - verification with determination of errors. The discriminative power of each of the parameters was evaluated by the Wilkes $\lambda$ index, which, in essence, is an analogue of the partial correlation.

In addition, we determined the weighting coefficients of the linear canonical discriminant function:

$$
y=\sum_{i=1}^{N} a_{1} \cdot x_{1}+a_{2} \cdot x_{2}+\ldots+a_{i} \cdot x_{i}+C,
$$

where $a_{i}$ is the weighting coefficient of the $i$-th parameter, $x_{i}$ is the value of the $i$-th parameter, and $C$ is a constant.

Statistical analysis. The obtained data were analyzed using the Statistica v. 10.0 software (StatSoft Inc., USA). The sample distribution mode was evaluated using the Shapiro-Wilk test. It was found that for most indicators the distribution deviated from normal $(p<0.01)$; therefore all descriptive statistics are presented as the median, $25^{\text {th }}$ and $75^{\text {th }}$ percentiles, maximum and minimum. For the pairwise comparison of the results, considering that the samples were dependent variables, we used the Wilcoxon W-test; the differences were considered significant at $p<0.05$.

\section{Results}

The general characteristics of patients before the TAVR procedure are presented in Table 1. All patients included in the study demonstrated a successful outcome without intra-hospital mortality or the need for repeated aortic valve replacement (Table 2).

Statistical processing. The analysis showed significant differences $(p<0.05)$ between the measurements taken before and after the intervention; these changes reflected the process of heart remodeling that was primarily expressed 
by changes in the left ventricular (LV) volume: relative wall thickness (RWT), IVS thickness, LVPW thickness, MM, SV, as well as Pmax and the ascending aorta diameter (Table 3). The statistical significance of these changes indicated positive dynamics in myocardial remodeling: a decrease in myocardial mass and wall thickness, i.e. a reduction in LV hypertrophy.

Discriminant analysis. According to our analysis, the risk of LBBB and the risk of paraprosthetic regurgitation after TAVR as predicted from the TTE parameters were similar. In the analysis, we identified the main parameters having the greatest power of prediction regarding these two complications (Table 4). In this table, the parameters are arranged in decreasing order of their impact on the prognostic function; the most contributing parameters were three anatomical characteristics of the left heart - IVS, LVPW, and LA, and the only functional characteristic - SV. The size of the transcatheter prosthesis, MM, and the surgical risk by EuroSCORE II had the lowest (although statistically significant) contribution to the prognostic function. The rest of the TTE parameters and the clinical and demographic characteristics of patients had no significant power of prediction.

Based on this selection, the weighting coefficients of the linear discriminant function were obtained (Table 5). These coefficients are the elements $a_{1}, a_{2}, a_{3}, a_{4}, a_{5}$, and $a_{6}$ of the canonical discriminant function. To calculate
Table 2

Intra- and postoperative interventions

\begin{tabular}{lc}
\hline \multicolumn{1}{c}{ Characteristic } & Value \\
\hline Artificial lung ventilation $(\mathrm{h})^{*}$ & $4.5[3.0 ; 3.5 ; 7.0 ; 7.5]$ \\
\hline Inotropic support & 0 \\
\hline Stay in the intensive care unit (days) & $1.0[1.0 ; 1.0 ; 2.0 ; 2.5]$ \\
\hline Duration of hospital stay (days) & $10.5[8.0 ; 8.0 ; 12.0 ; 13.0]$ \\
\hline Successful procedures (abs. number/\%)\# & $10 / 100$ \\
\hline Intra-hospital mortality & 0 \\
\hline Repeated procedure & 0 \\
\hline Paraprosthetic regurgitation, grade $\geq \| I$ (abs. number/\%) & $3 / 30$ \\
\hline Left bundle branch block (abs. number/\%) & $4 / 40$ \\
\hline Myocardial infarction & 0 \\
\hline Hydrothorax (abs. number/\%) & $1 / 10$ \\
\hline Atrial fibrillation & 0 \\
\hline Pacemaker implantation & 0 \\
\hline Vascular access complications & 0 \\
\hline
\end{tabular}

* Data are presented as Me [min; 25; 75; max]; \# according to the criteria of the Valve Academic Research Consortium-2 [8].

the risk of $\angle B B B$ or paraprosthetic regurgitation, one has to multiply the weighting coefficients $a_{1}-a_{6}$ in pairs by the parameter values obtained from the preoperative TTE (based on Table 5), and then put the result into the indicated equation. If the final value is less than zero, one can predict the occurrence of complications and, conversely, with a positive final value - no block or regurgitation is expected.

Table 3

Heart remodeling indicators obtained by transthoracic echocardiography

\begin{tabular}{|c|c|c|c|c|c|c|c|c|c|c|c|}
\hline \multirow{2}{*}{ Parameters } & \multicolumn{5}{|c|}{ Before TAVR } & \multicolumn{5}{|c|}{ After TAVR } & \multirow{2}{*}{$p$} \\
\hline & Me & Min & Max & $25 \%$ & $75 \%$ & $\mathrm{Me}$ & Min & Max & $25 \%$ & $75 \%$ & \\
\hline End-diastolic diameter & 5.7 & 4.7 & 6.9 & 5.2 & 6.1 & 5.7 & 4.7 & 6.7 & 5.1 & 6.3 & 0.86 \\
\hline End-systolic diameter & 3.7 & 3.0 & 5.0 & 3.3 & 3.8 & 3.7 & 3.0 & 5.6 & 3.3 & 4.1 & 0.26 \\
\hline Ejection fraction & 63.0 & 52.0 & 71.0 & 54.0 & 70.0 & 63.0 & 33.0 & 73.0 & 45.0 & 66.0 & 0.44 \\
\hline End-diastolic volume & 157.0 & 102.0 & 247.0 & 130.0 & 187.0 & 157.0 & 102.0 & 231.0 & 124.0 & 201.0 & 0.74 \\
\hline End-systolic volume & 56.5 & 35.0 & 118.0 & 41.0 & 62.0 & 56.5 & 35.0 & 154.0 & 44.0 & 74.0 & 0.22 \\
\hline Left ventricular relative wall thickness & 0.44 & 0.38 & 0.40 & 0.49 & 0.60 & 0.43 & 0.33 & 0.38 & 0.48 & 0.55 & 0.05 \\
\hline Left atrium dimension & 4.7 & 4.1 & 5.8 & 4.4 & 4.9 & 4.6 & 3.7 & 6.3 & 4.5 & 5.0 & 0.95 \\
\hline Right ventricular dimension & 1.9 & 1.6 & 2.8 & 1.8 & 2.1 & 2.0 & 1.7 & 3.1 & 1.8 & 2.2 & 0.22 \\
\hline Interventricular septal thickness & 1.4 & 1.1 & 1.6 & 1.3 & 1.5 & 1.3 & 1.0 & 1.5 & 1.2 & 1.4 & 0.05 \\
\hline Left ventricular posterior wall thickness & 1.4 & 1.1 & 1.6 & 1.3 & 1.5 & 1.3 & 1.0 & 1.4 & 1.2 & 1.3 & 0.05 \\
\hline Ascending aorta diameter & 3.6 & 3.0 & 4.3 & 3.3 & 4.1 & 3.4 & 2.6 & 4.0 & 3.0 & 3.5 & 0.02 \\
\hline Stroke volume & 107.5 & 64.0 & 129.0 & 79.0 & 122.0 & 92.0 & 52.0 & 127.0 & 77.0 & 105.0 & 0.33 \\
\hline Myocardial mass & 342.0 & 261.0 & 497.0 & 322.0 & 421.0 & 337.5 & 200.0 & 409.0 & 237.0 & 367.0 & 0.05 \\
\hline Maximum trans-prosthesis gradient & 77.5 & 65.0 & 115.0 & 70.0 & 93.0 & 11.5 & 7.0 & 17.0 & 11.0 & 16.0 & 0.01 \\
\hline
\end{tabular}

Note s: statistically significant differences between the measurements made before and after TAVR are shown in bold. The indicator of the relative thickness of the LV walls is estimated as (LVPW thickness + IVS thickness)/EDD. 


\section{ADVANCED RESEARCHES}

Table 4

Key discriminative parameters of the post-TAVR complications

\begin{tabular}{|c|c|c|c|}
\hline \multicolumn{2}{|l|}{ Left bundle branch block } & \multicolumn{2}{|c|}{ Paraprosthetic regurgitation } \\
\hline Parameters & Wilkes $\Lambda$ & Parameters & Wilkes $\Lambda$ \\
\hline Left ventricular posterior wall thickness & 0.322 & Stroke volume & 0.076 \\
\hline Stroke volume & 0.210 & Right ventricular dimension & 0.043 \\
\hline Interventricular septal thickness & 0.288 & Left ventricular posterior wall thickness & 0.013 \\
\hline Left atrium dimension & 0.144 & Ascending aorta diameter & 0.089 \\
\hline Prosthesis size & 0.107 & Prosthesis size & 0.019 \\
\hline EuroSCORE II surgical risk & 0.011 & Myocardial mass & 0.004 \\
\hline Mahalanobis distance & 744.72 & Mahalanobis distance & 4890.33 \\
\hline$p$-value related to the entire function & 0.01 & $p$-value related to the entire function & 0.01 \\
\hline Classification errors (\%) & 10.0 & Classification errors (\%) & 0 \\
\hline
\end{tabular}

Table 5

Linear discriminative function coefficients

\begin{tabular}{|c|c|c|c|c|c|}
\hline \multicolumn{3}{|c|}{ Left bundle branch block } & \multicolumn{3}{|c|}{ Paraprosthetic regurgitation } \\
\hline Parameters & $\begin{array}{l}\text { Weighting } \\
\text { coefficient value }\end{array}$ & $\begin{array}{l}\text { Weighting } \\
\text { coefficient } \\
\text { number }\end{array}$ & Parameters & $\begin{array}{l}\text { Weighting } \\
\text { coefficient value }\end{array}$ & $\begin{array}{c}\text { Weighting } \\
\text { coefficient } \\
\text { number }\end{array}$ \\
\hline Left ventricular posterior wall thickness & $23,672.6$ & $a_{1}$ & Stroke volume & 6.711 & $a_{1}$ \\
\hline Stroke volume & -15.2 & $a_{2}$ & Right ventricular dimension & 122.174 & $a_{2}$ \\
\hline Interventricular septal thickness & $-19,506.7$ & $a_{3}$ & Left ventricular posterior wall thickness & -917.906 & $a_{3}$ \\
\hline Left atrium dimension & 945.2 & $a_{4}$ & Ascending aorta diameter & 306.685 & $a_{4}$ \\
\hline Prosthesis size & -252.7 & $a_{5}$ & Prosthesis size & -49.983 & $a_{5}$ \\
\hline EuroSCORE II surgical risk & -28.6 & $a_{6}$ & Myocardial mass & 0.258 & $a_{6}$ \\
\hline Constant & -976.2 & C & Constant & 372.771 & C \\
\hline
\end{tabular}

N o t e: the number of the weighting coefficient is given for the canonical form of the discriminant function.

\section{Discussion}

Statistical analysis. The pair-wise statistical comparison showed significant differences in LV myocardial parameters between the measurements made before and after the TAVR procedure: those related to IVS, LVPW, and MM. Along with that, almost all LV volume parameters (EDV, ESV, EF, etc.) did not significantly change after the intervention. The reason for these differences may lie in the nature of the aortic defect, i.e., stenosis without hemodynamically significant regurgitation. This pathology leads to the development of compensatory concentric hypertrophy, associated primarily with thickening of the myocardial wall without significant changes in the heart cavities volume. The RWT indicator precisely confirms the concentric model - in this study, the median RWT value was 0.44 at a threshold value of 0.42 , which indicated a significant increase in the thickness of LV walls relative to the cavity volume. After the TAVR procedure, the cause of hemodynamic disturbances - valve stenosis with a high peak gradient - is eliminated; the resulting heart remodeling reduces the myocardial mass, but barely changes the dimensions of the heart cavities, which initially were close to normal $(60-130 \mathrm{~mm})$. It is noteworthy that such an effect can occur only if two conditions are met: 1) the initial stenosis of the aortic valve does not cause regurgitation and 2) the valve defect is functionally compensated. Condition 1 is associated with concentric hypertrophy, i.e., a change in the mass, but not the volume of the left ventricle; condition 2 is determined by the compensatory mechanism, where a significant increase in LV volume (development of eccentric hypertrophy) is possible only after a compensation "failure". In the present study, both conditions were present in all patients, which contributed to the statistical significance of the results (see Table 3).

Our results are in good agreement with similar works by other authors. Thus, a wide range of TTE parameters measured before and after TAVR (Stangl et al. [9]), showed significant changes in IVS, LVPW, and $\mathrm{MM}$ - and no changes in volume indicators - EF (only 
for men) and EDD. In a study of Rymuza et al. [10] in patients with the RWT of $0.6 \pm 0.1 \mathrm{~cm}$, EDD, EDV, and EF did not differ between the pre- and postoperative periods (12 months) due to the same reasons - the initial presence of both concentric and compensated hypertrophy.

Discriminant analysis. Our discriminant analysis revealed a significant contribution of anatomical indicators to the risk of both complications - LBBB and regurgitation, which, apparently, requires a more thorough analysis of these phenomena. In the current practice of preoperative planning, it is recommended (2017 ESC/EACTS guidelines) to use MSCT to assess the anatomy and size of the aortic root, the shape and size of the valve annulus, its distance to the mouth of the coronary arteries, the distribution of calcifications and the number of aortic valve cusps [1]. In addition, according to this recommendation, MSCT is more preferable than TTE because the latter highly depends on the operator and may also have sub-optimal image quality. However, the present study shows that the results of TTE, which primarily reflect the anatomy of the left heart (LVPW, IVS, LA), also have important prognostic values.

It is noteworthy that, in addition to the anatomical characteristics, the size of the prosthesis for TAVR also contributed to the prediction algorithm. The development of LBBB is caused by excessive pressure exerted by the supporting frame of a self-expanding prosthesis on the conduction paths passing near the fibrous ring of the aortic valve [11]. In this study, a significant contribution of the prosthesis size to the risk of both complications was found. The result necessitates a more careful selection of bio-prostheses for implantation, including the new version of Evolut $R^{\mathrm{TM}}$ (Medtronic Inc., USA) [12].

In paraprosthetic regurgitation, the mechanism is associated with severe valvular calcification, which prevents the prosthesis from full straightening and tightfitting [13]. Presumably, a smaller prosthesis would produce less force than that needed to shift the calcium deposits to the periphery. Nevertheless, despite its significance the contribution of the size indicator to the final prognostic model is rather small (see Table 4) and should be interpreted with caution.

In general, the obtained linear function coefficients (see Table 5) demonstrate a high power of prediction for LBBB and regurgitation risk. However, we found that in the case of LBBB, the coefficient values had a $10 \%$ error, which, according to the statistical analysis (classification matrix) produced false-positive estimates. On the one hand, the presence of such an error can be considered non-critical, since for this type of intervention a false-positive result can be interpreted as additional "insurance" - the model predicts the risk of complications, although in practice it may not happen. On the other hand, the mere presence of a classification error is a negative factor, which does not allow us to definitely recommend this algorithm for decision-making at the preoperative stage. Additionally, it is worth noting that in the present study, the number of patients was limited but uniform in terms of its clinical characteristics (demography, functions, comorbidity). Therefore, the obtained coefficients of the linear function are somewhat "idealized" and may prove less sensitivity/specificity for more heterogeneous groups.

\section{Conclusion}

Statistically significant changes in morphology and function of the left ventricle (primarily, thickness and mass of the myocardium) indicating heart remodeling after transcatheter aortic valve replacement were demonstrated. The obtained discriminative linear function allows one to predict the risk of two major complications of transcatheter aortic valve replacement - left bundle branch block and paraprosthetic regurgitation $(p<0.01)$. This conclusion, although based on the parameters of heart anatomy, should be taken with caution, since (in this study) it produced $10 \%$ of false-positive predictions for left bundle branch block.

Research funding. This work was supported by a grant from the Russian Science Foundation (project 1875-10061) for a project "Research and implementation of the concept of robotic minimally invasive aortic valve replacement".

Conflict of interest. The authors declare no conflict of interest.

\section{References}

1. Baumgartner H., Falk V., Bax J.J., De Bonis M., Hamm C., Holm P.J., lung B., Lancellotti P., Lansac E., Rodriguez Muñoz D., Rosenhek R., Sjögren J., Tornos Mas P., Vahanian A., Walther T., Wendler O., Windecker S., Zamorano J.L.; ESC Scientific Document Group. 2017 ESC/ EACTS guidelines for the management of valvular heart disease. Eur Heart J 2017; 38(36): 2739-2791, https://doi. org/10.1093/eurheartj/ehx391.

2. Leon M.B., Smith C.R., Mack M.J., Makkar R.R., Svensson L.G., Kodali S.K., Thourani V.H., Tuzcu E.M., Miller D.C., Herrmann H.C., Doshi D., Cohen D.J., Pichard A.D., Kapadia S., Dewey T., Babaliaros V., Szeto W.Y., Williams M.R., Kereiakes D., Zajarias A., Greason K.L., Whisenant B.K., Hodson R.W., Moses J.W., Trento A., Brown D.L., Fearon W.F., Pibarot P., Hahn R.T., Jaber W.A., Anderson W.N., Alu M.C., Webb J.G.; PARTNER 2 Investigators. Transcatheter or surgical aortic-valve replacement in intermediate-risk patients. N Engl J Med 2016; 374(17): 1609-1620, https://doi.org/10.1056/nejmoa1514616.

3. Reardon M.J., Van Mieghem N.M., Popma J.J., Kleiman N.S., Søndergaard L., Mumtaz M., Adams D.H., Deeb G.M., Maini B., Gada H., Chetcuti S., Gleason T., Heiser J., Lange R., Merhi W., Oh J.K., Olsen P.S., Piazza N., Williams M., Windecker S., Yakubov S.J., Grube E., Makkar R., Lee J.S., Conte J., Vang E., Nguyen H., Chang Y., Mugglin A.S., Serruys P.W., Kappetein A.P.; SURTAVI Investigators. Surgical or transcatheter aortic-valve replacement in intermediate-risk 
patients. N Engl J Med 2017; 376(14): 1321-1331, https://doi. org/10.1056/nejmoa1700456.

4. Thyregod H.G., Steinbrüchel D.A., Ihlemann N., Nissen H., Kjeldsen B.J., Petursson P., Chang Y., Franzen O.W., Engstrøm T., Clemmensen P., Hansen P.B., Andersen L.W., Olsen P.S., Søndergaard L. Transcatheter versus surgical aortic valve replacement in patients with severe aortic valve stenosis: 1-year results from the all-comers NOTION randomized clinical trial. J Am Coll Cardiol 2015 65(20): 2184-2194, https://doi.org/10.1016/j.jacc.2015.03.014.

5. Siu S.C., Silversides C.K. Bicuspid aortic valve disease. J Am Coll Cardiol 2010; 55(25): 2789-2800, https://doi. org/10.1016/j.jacc.2009.12.068.

6. Yoon S.H., Bleiziffer S., De Backer O., Delgado V., Arai T., Ziegelmueller J., Barbanti M., Sharma R., Perlman G.Y., Khalique O.K., Holy E.W., Saraf S., Deuschl F., Fujita B., Ruile P., Neumann F.J., Pache G., Takahashi M., Kaneko H., Schmidt T., Ohno Y., Schofer N., Kong W.K.F., Tay E., Sugiyama D., Kawamori H., Maeno Y., Abramowitz Y., Chakravarty T., Nakamura M., Kuwata S., Yong G., Kao H.L., Lee M., Kim H.S., Modine T., Wong S.C., Bedgoni F., Testa L., Teiger E., Butter C., Ensminger S.M., Schaefer U., Dvir D., Blanke P., Leipsic J., Nietlispach F., Abdel-Wahab M., Chevalier B., Tamburino C., Hildick-Smith D., Whisenant B.K., Park S.J., Colombo A., Latib A., Kodali S.K., Bax J.J., Søndergaard L., Webb J.G., Lefèvre T., Leon M.B., Makkar R. Outcomes in transcatheter aortic valve replacement for bicuspid versus tricuspid aortic valve stenosis. J Am Coll Cardiol 2017; 69(21): 2579-2589, https://doi.org/10.1016/j. jacc.2017.03.017.

7. Neylon A., Ahmed K., Mercanti F., Sharif F., Mylotte D. Transcatheter aortic valve implantation: status update. J Thorac Dis 2018; 10(Suppl 30): S3637-S3645, https://doi. org/10.21037/jtd.2018.10.34.

8. Kappetein A.P., Head S.J., Généreux P., Piazza N., van Mieghem N.M., Blackstone E.H., Brott T.G., Cohen D.J., Cutlip D.E., van Es G.A., Hahn R.T., Kirtane A.J., Krucoff M.W., Kodali S., Mack M.J., Mehran R., RodésCabau J., Vranckx P., Webb J.G., Windecker S., Serruys P.W.,
Leon M.B.; Valve Academic Research Consortium (VARC)-2. Updated standardized endpoint definitions for transcatheter aortic valve implantation: the Valve Academic Research Consortium-2 consensus document (VARC-2). Eur J Cardiothorac Surg 2012; 42(5): S45-S60, https://doi. org/10.1093/ejcts/ezs533.

9. Stangl V., Baldenhofer G., Knebel F., Zhang K., Sanad W., Spethmann S., Grubitzsch H., Sander M., Wernecke K.D., Baumann G., Stangl K., Laule M. Impact of gender on three-month outcome and left ventricular remodeling after transfemoral transcatheter aortic valve implantation. Am $J$ Cardiol 2012; 110(6): 884-890, https://doi.org/10.1016/j. amjcard.2012.04.063.

10. Rymuza B., Zbroński K., Scisło P., Wilimski R., Kochman J., Ćwiek A., Filipiak K.J., Opolski G., Huczek Z. Left ventricular remodelling pattern and its relation to clinical outcomes in patients with severe aortic stenosis treated with transcatheter aortic valve implantation. Postepy Kardiol Interwencyjnej 2017; 13(4): 288-294, https://doi.org/10.5114/ aic. 2017.71609.

11. Houthuizen P., Van Garsse L.A., Poels T.T., de Jaegere P., van der Boon R.M., Swinkels B.M. Ten Berg J.M., van der Kley F., Schalij M.J., Baan J. Jr., Cocchieri R., Brueren G.R., van Straten A.H., den Heijer P., Bentala M., van Ommen V., Kluin J., Stella P.R., Prins M.H., Maessen J.G., Prinzen F.W. Left bundle-branch block induced by transcatheter aortic valve implantation increases risk of death. Circulation 2012; 126(6): 720-728, https://doi. org/10.1161/circulationaha.112.101055.

12. Schulz E., Jabs A., Gori T., von Bardeleben S., Hink U., Kasper-König W., Vahl C.F., Münzel T. Transcatheter aortic valve implantation with the new-generation Evolut $R^{\mathrm{TM}}$ : comparison with CoreValve ${ }^{\circledR}$ in a single center cohort. Int J Cardiol Heart Vasc 2016; 12: 52-56, https://doi.org/10.1016/j. ijcha.2016.06.002.

13. Wilczek K., Bujak K., Reguła R., Chodór P., Osadnik T. Risk factors for paravalvular leak after transcatheter aortic valve implantation. Kardiochir Torakochirurgia Pol 2015; 12(2): 89-94, https://doi.org/10.5114/kitp.2015.52848. 